\title{
Underwater endoscopic submucosal dissection of a nonpolypoid superficial tumor spreading into the appendix
}

Federico Iacopini, MD, ${ }^{1}$ Takuji Gotoda, PhD, FASGE, ${ }^{2}$ Fabrizio Montagnese, MD,${ }^{1}$ Fabio Andrei, MD, ${ }^{1}$ Yutaka Saito, $\mathbf{M D}, \mathbf{P h D}^{3}$

Superficial tumors at the appendix are mostly off limits for endoscopic resection due to the technical challenge of a complete resection and risk of perforation because of the geometrically difficult anatomy. ${ }^{1}$

A 72-year-old man underwent colonoscopy with the diagnosis of a granular laterally spreading tumor invading the appendix from the cecum. The tumor had irregular capillary (type 2B, JNET classification) and tubular pit patterns (type IIIL, Kudo classification). The tip of the colonoscope was pushed onto the appendiceal orifice, and a $20-\mathrm{mm}$ tumor spreading into the appendix for half of its area was visualized.

Endoscopic submucosal dissection (ESD) was scheduled with a pediatric colonoscope (PCF-H180AI, Olympus, Tokyo, Japan) and a transparent hood with a short $(7 \mathrm{~mm})$ small-caliber $(8 \mathrm{~mm})$ tapered tip (SThood, Fujifilm, Tokyo, Japan). The appendix was intubated for a few millimeters. Water irrigation rather than air inflation was used to avoid pain and maintain a steady distension (Fig. 1).

A mixture of hydroxyethyl starch (Voluven, Fresenius Kabi, Isola della Scala, Italy), epinephrine (1:250.000), and indigo carmine was used for submucosal injection. The electrosurgical VIO200 generator (ERBE, Tubingen, Germany) was set at endocut I (effect 2, duration 3 , interval 1) for mucosal incision and swift coagulation (effect 4, $40 \mathrm{~W}$ ) for submucosal dissection and hemostasis. The mucosal incision started from the appendiceal edge of the neoplasm (Fig. 2). Submucosal dissection was conducted from the cecum to the appendix with a $1.5-\mathrm{mm}$ knife (Dualknife, KD-650U, Olympus) and a small insulated-tip knife (IT-knife nano, Olympus) (Fig. 3A).

Submucosal fibrosis was severe and diffuse (Fig. 3B). ESD was completed en bloc within 107 minutes (Fig. 4, Video 1, available online at www.VideoGIE.org). Ciprofloxacin (500 $\mathrm{mg}$ twice daily) was initiated at the end of the procedure and maintained for 3 days. Fever $\left(38^{\circ} \mathrm{C}\right)$, mild pain, and rebound tenderness in the right ileal fossa occurred after 8 hours and resolved conservatively within the following 24 hours. Histologic examination demonstrated the curative resection of an adenoma with high-grade dysplasia. The 6month and 12-month follow-up examinations showed a flat scar and no stricture (Fig. 5).

Tumor extension to the appendix can be limited to the orifice (type 1) or may spread into the lumen with visible (type 2 ) or invisible (type 3 ) margins. ${ }^{2,3}$ Visible

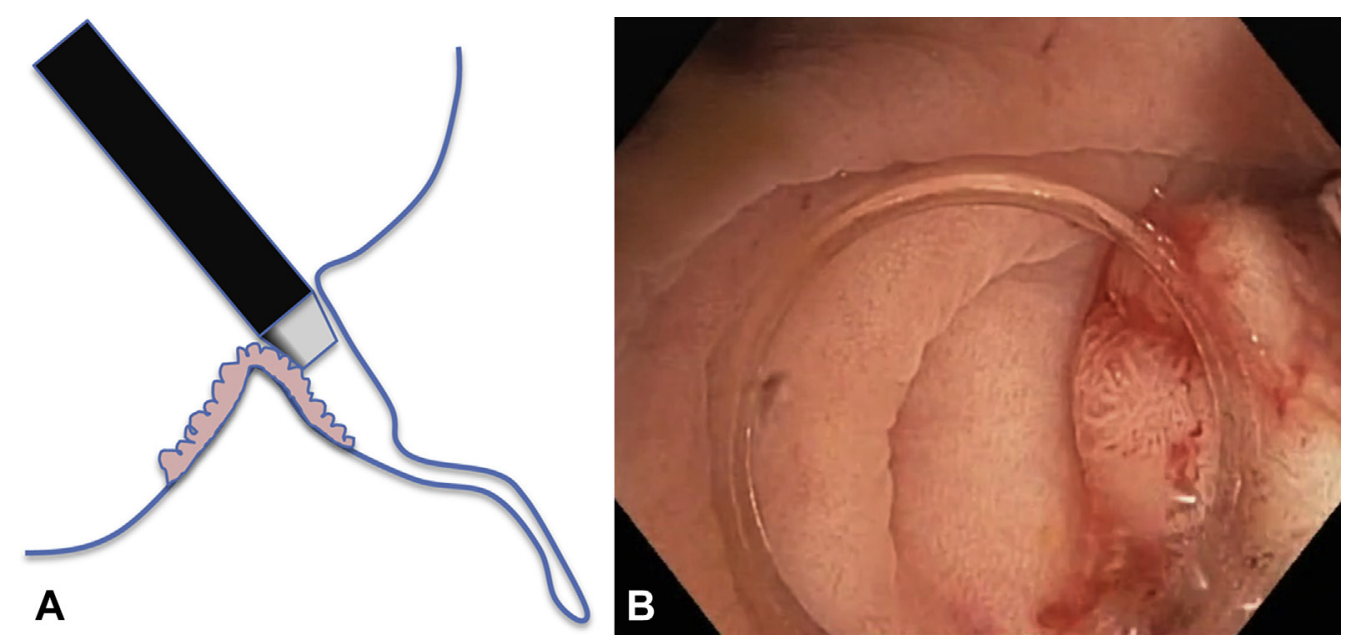

Figure 1. A, Underwater intubation of the appendix. B, Visualization of the appendiceal tumor margins. 

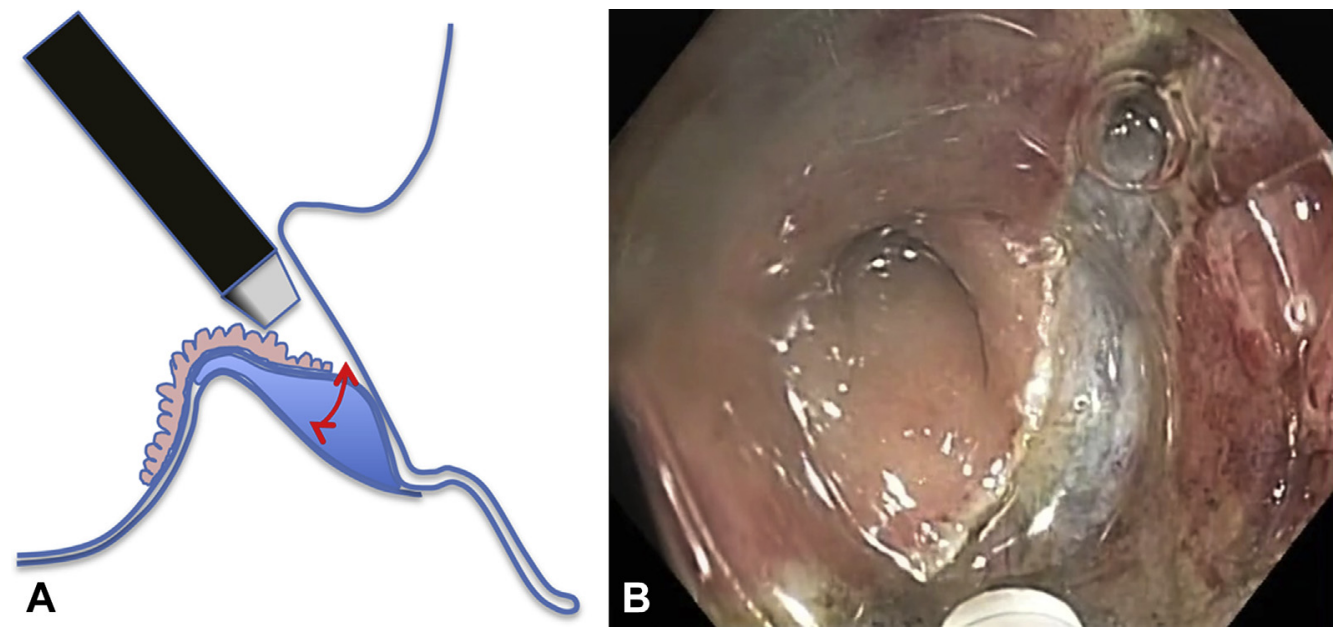

Figure 2. A, Small-volume submucosal injection at the appendiceal tumor edge (red arrow) avoiding lumen occlusion and lifting at the orifice with $\mathbf{B}$, subsequent mucosal incision.
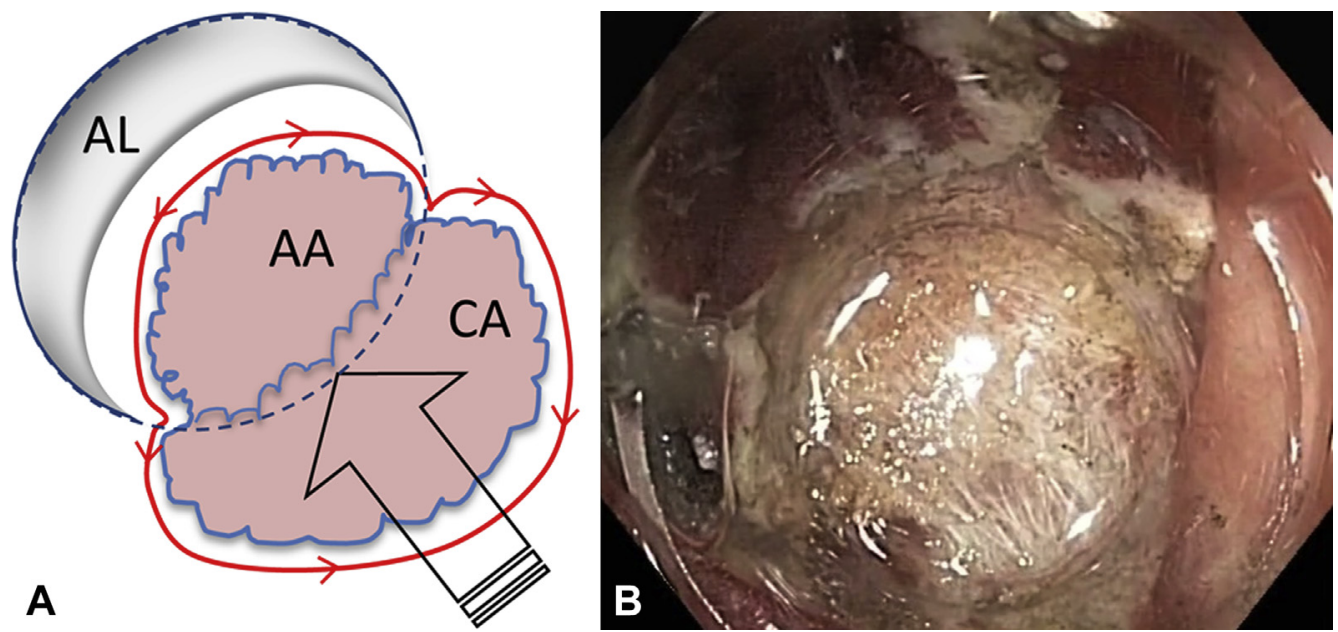

Figure 3. A, Retrograde circumferential mucosal incision (red arrows) and straightforward dissection from the cecal area (CA) to the appendiceal area (AA) (black arrow) into the appendiceal lumen (AL). B, Severe submucosal fibrosis.

and reachable margins are prerequisites for the feasibility of endoscopic resection. In published reports, 39 tumors spreading into the appendix have undergone endoscopic resection (Table 1). Most cases underwent underwater EMR or ESD after a partial invagination of the appendix and tumor prolapse into the cecum with a $10 \%$ and $0 \%$ recurrence rate, respectively. Specific adverse events were early and late acute appendicitis, in 3\% and 5\% of cases, respectively, and post-polypectomy syndrome occurred in $10 \%$.

This video proposes a new ESD approach based on appendiceal intubation and underwater technique, which guaranteed a stable position and luminal distension, avoidance of pain, and possible reduction of the risk of perforation. The ESD sequential cut was analogous to that standardized for the esophagus ${ }^{9}$ : mucosal incision started from the distal edge, whereas dissection proceeded in a straightforward manner. Preoperative peripheral markings were not necessary because colorectal tumor margins are generally clearly visible. ESD was preferred because of the deep spreading of the tumor into the appendix. The abrupt angulation at the transition between the cecum and appendix, the limited appendiceal space, and the prevalence of submucosal fibrosis that rises with decreasing distance from the appendiceal orifice ${ }^{2}$ predict impaired and blind snare maneuvers with a high risk of piecemeal or incomplete resection. Appendiceal ESD seems feasible, and manipulation of knives very close to the tumor may guarantee higher rates of en bloc and complete resection, but endoscopists should be familiar with submucosal fibrosis and counter traction methods. ${ }^{10}$

\section{DISCLOSURE}

All authors disclosed no financial relationships relevant to this publication.

Abbreviation: ESD, endoscopic submucosal dissection. 


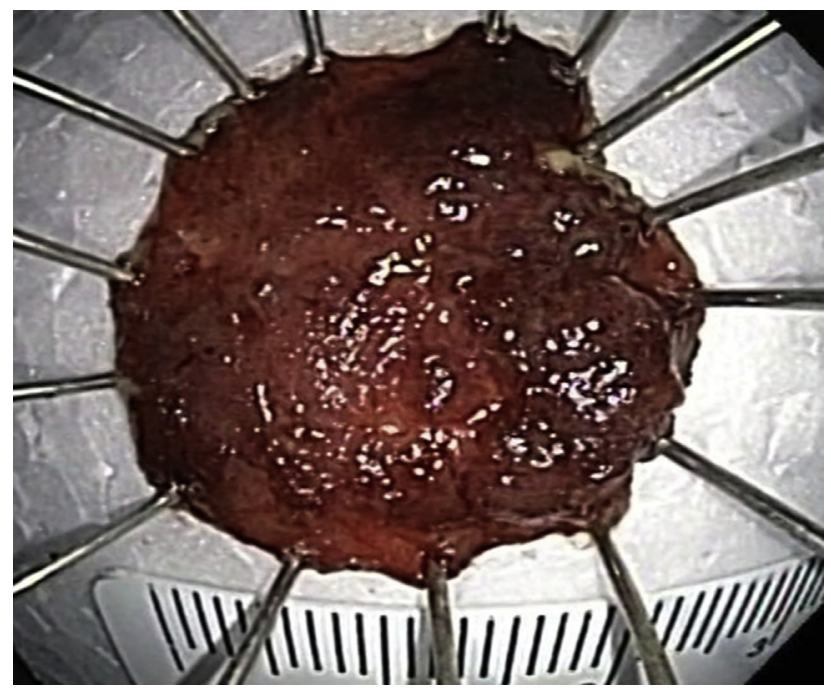

Figure 4. Resected specimen.

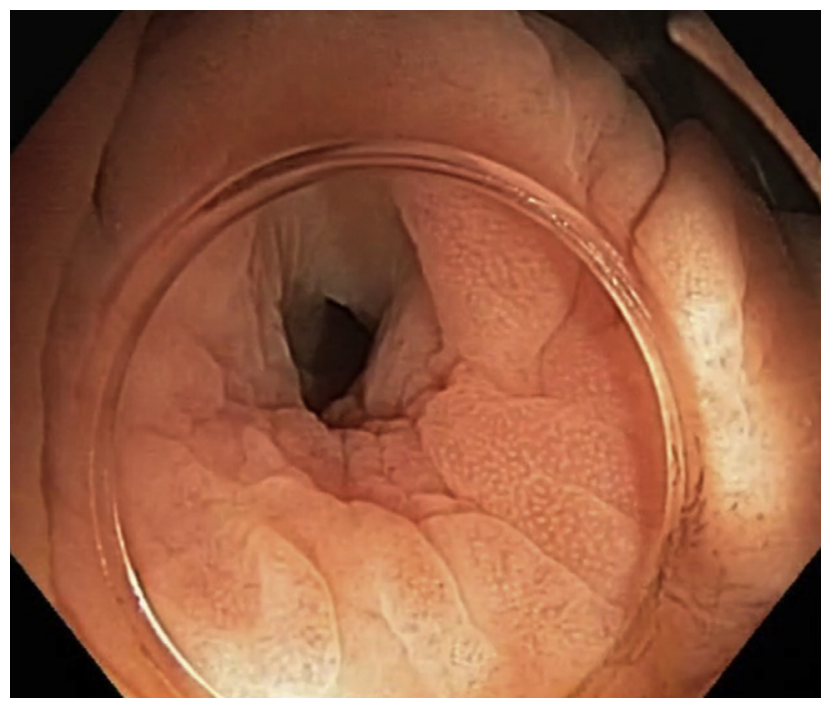

Figure 5. Scar at the resection site.

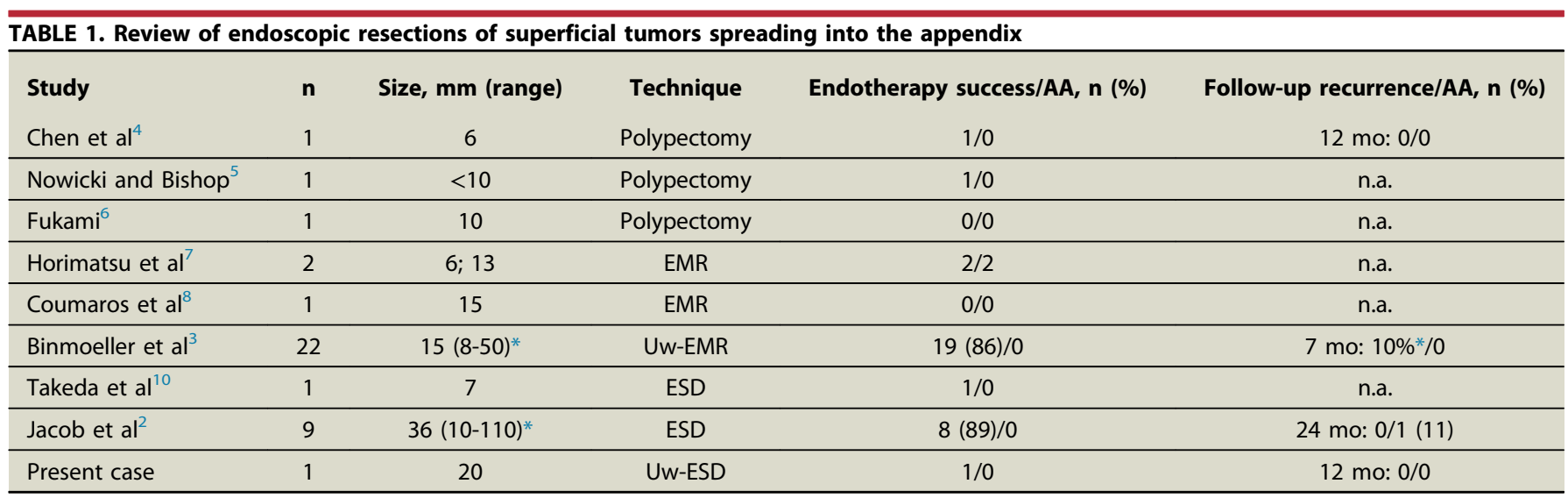

$A A$, Acute appendicitis; $E R$, endoscopic resection; $E S D$, endoscopic submucosal dissection; mo, months; n.a., not available; Uw, underwater.

*Also comprising tumors at the appendiceal orifice.

\section{REFERENCES}

1. Sakamoto I, Watanabe S, Sakuma T, et al. Intramucosal adenocarcinoma of the appendix: how to find and how to treat. Endoscopy 2003;35:785-7.

2. Jacob H, Toyonaga T, Ohara Y, et al. Endoscopic submucosal dissection of cecal lesions in proximity to the appendiceal orifice. Endoscopy 2016;48: 829-36.

3. Binmoeller KF, Hamerski CM, Shah JN, et al. Underwater EMR of adenomas of the appendiceal orifice (with video). Gastrointest Endosc 2016;83:638-42.

4. Chen YY, Soon MS, Yen HH. Gastrointestinal: adenomatous polyp of the appendix. J Gastroenterol Hepatol 2006;21:617.

5. Nowicki MJ, Bishop PR. Successful endoscopic removal of an appendiceal polyp in a child with juvenile polyposis syndrome. Gastrointest Endosc 2011;74:441-3.

6. Fukami $\mathrm{Y}$, Hasagawa $\mathrm{H}$, Ogiso $\mathrm{S}$, et al. A case of adenoma of the appendix diagnosed preoperatively. J J.jpn Surg Assoc 2001;62:2215-8.

7. Horimatsu T, Ki Fu, Sano Y, et al. Acute appendicitis as a rare complication after endoscopic mucosal resection. Dig Dis Sci 2007;52:1741-4.

8. Coumaros D, Mavorgenis G, Anselm Y, et al. "Volcano sign" and endoscopic mucosal resection of a villous adenoma arising from the appendix. Dig Liver Dis 2012;44:e13.
9. Arantes V, Albuquerque W, Freitas Dias CA, et al. Standardized endoscopic submucosal tunnel dissection for management of early esophageal tumors (with video). Gastrointest Endosc 2013;78: 946-52.

10. Takeda T, Murakami T, Sakamoto N, et al. Traction device to remove an adenoma in the appendiceal orifice by endoscopic submucosal dissection. Endoscopy 2013;45:E239-40.

Gastroenterology and Endoscopy Unit, Ospedale S. Giuseppe, Rome, Italy (1), Division of Gastroenterology and Hepatology, Nihon University School of Medicine, Tokyo, Japan (2), Endoscopy Division, National Cancer Center Hospital, Tokyo, Japan (3).

Copyright (c) 2017 American Society for Gastrointestinal Endoscopy. Published by Elsevier Inc. This is an open access article under the CC BYNC-ND license (http://creativecommons.org/licenses/by-nc-nd/4.0/).

http://dx.doi.org/10.1016/j.vgie.2017.01.007 